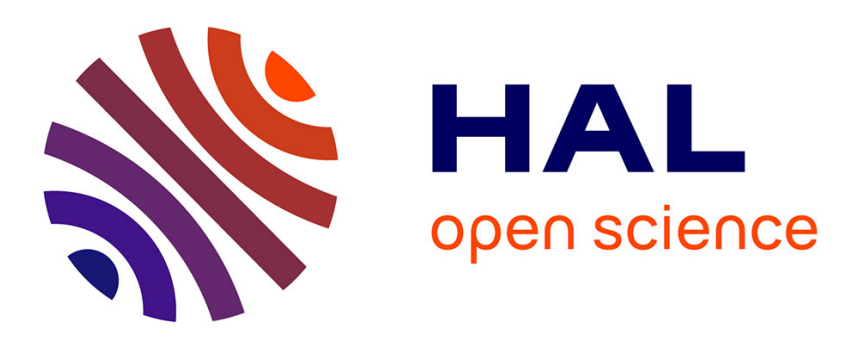

\title{
Application of PIL Approach for Automated Transportation Center
}

Stepan Ozana, Martin Pies, Radovan Hajovsky, Jiri Koziorek, Ondrej Horacek

\section{To cite this version:}

Stepan Ozana, Martin Pies, Radovan Hajovsky, Jiri Koziorek, Ondrej Horacek. Application of PIL Approach for Automated Transportation Center. 13th IFIP International Conference on Computer Information Systems and Industrial Management (CISIM), Nov 2014, Ho Chi Minh City, Vietnam. pp.501-513, 10.1007/978-3-662-45237-0_46 . hal-01405633

\section{HAL Id: hal-01405633 \\ https://hal.inria.fr/hal-01405633}

Submitted on 30 Nov 2016

HAL is a multi-disciplinary open access archive for the deposit and dissemination of scientific research documents, whether they are published or not. The documents may come from teaching and research institutions in France or abroad, or from public or private research centers.
L'archive ouverte pluridisciplinaire HAL, est destinée au dépôt et à la diffusion de documents scientifiques de niveau recherche, publiés ou non, émanant des établissements d'enseignement et de recherche français ou étrangers, des laboratoires publics ou privés.

\section{(c)(1)}

Distributed under a Creative Commons Attribution| 4.0 International License 


\title{
Application of PIL approach for Automated Transportation Center
}

\author{
Stepan Ozana, Martin Pies, Radovan Hajovsky, Jiri Koziorek, and \\ Ondrej Horacek \\ VSB-Technical university of Ostrava, \\ Faculty of Electrical Engineering and Computer Science, \\ Department of Cybernetics and Biomedical Engineering, \\ 17. listopadu 15/2172, 70833 Ostrava, Czech Republic \\ \{stepan.ozana, martin.pies, radovan.hajovsky, jiri.koziorek, ondrej.horacek\}@ \\ $\mathrm{vsb} . \mathrm{cz}$ \\ http://www.fei.vsb.cz/en
}

\begin{abstract}
The paper describes the idea of design and implementation of Processor-in-the-loop (PIL) model of Automated Transportation Center (ATC) designed as a heavy laboratory operated by VSB-Technical University of Ostrava. It contains technical description of the real technology, the main concept of PIL model and its particular solution for ATC. As for means of design and implementation, the REX Control system was used as a real-time target running on ALIX system board that handles the software model of real technology while the real control system based on Simatic PLCs stays unchanged. The communication between PLCs and ALIX is provided by PROFINET protocol while all of the original I/O signals are re-mapped to match the PROFINET communication standard.
\end{abstract}

Keywords: Automation, PIL, PLC, SCADA/HMI, Transportation

\section{Introduction}

This article gives an overview of control design and technical solution of PIL model for particular Automated Transportation Center but the principle of this solution can be easily applied in a wider scale to match various general technology complex units.

The main benefit of the paper and the project is to provide a tool for complex testing of real control systems without connecting to real technology.

During the construction of the new building of the Faculty of Electrical Engineering and Computer Science at VSB - Technical University of Ostrava (VSBTUO) with financial support of European Union, a unique heavy laboratory Automated Transportation Center (ATC) - was built. It is an absolutely rare real mechatronic system representing a fully automated pallet stacker machine that is able to reach four floors to store, for example, cars on pallets [1]. 
Within a single building, ATC deals with a connection between theory and practice - the research and development of security software and the real verification of the system of the automated, computer-controlled operation of the multi-story capacitive stacker machine with a direct transfer of $\mathrm{R} \& \mathrm{D}$ results to the application area.

The parcel designated for ATC - a square-shaped building with a space for a lab intended for teaching students on the ground floor, with technical facilities, four storage spaces and three other storage floors, and an automatic storage system with a total capacity of 37 cells - is situated on a free, unused area of the premises of VSB where, according to an area future development urban study, parking spaces are to be concentrated to eliminate the movement of motor vehicles within the university's large-scale campus. Fig. 1 shows a view of Automated Transportation Center located in VSB-TU Ostrava campus.



Fig. 1. A view of the Automated Transportation Center in Ostrava

Currently, when working on theses, students learn the lab's technology and their teachers participate in the further development and experimental verification of new features of the technology. The main usefulness of this infrastructure can be seen in the following points:

Faculty vehicles used for the purpose of educational and research activities can be safely stored here. Many of these vehicles were obtained as a gift from Hyundai Motor Manufacturing Czech in Nosovice and serve students as an educational tool. Other vehicles are a result of the faculty's own development activities, for example, Kaipan electric-drive cars. In ATC, these vehicles are protected against both weather conditions and vandalism.

It is a complex real mechatronic system equipped with a modern distributed control system, which demonstrates real issues associated with the operation of such a system. During lessons, when laboratory simulations are mostly performed, this can be seen as a great benefit and a unique experience for students.

The lab, which is located on the first floor, allows monitoring, analyzing, and storage of real-time data from the system's operation. Students can see this 
operation with their own eyes and become much more aware of potential risks related to errors in the control of such systems.

In the future, the concept of the automated parking system, which is the technological heart of the laboratory, could be used in densely populated urban agglomerations. Today, there are more and more places where such systems are being used. This laboratory allows teachers and students to participate in its further development.

In the future, the concept of the automated parking system, which is the technological heart of the laboratory, could be used in densely populated urban agglomerations. Today, there are more and more places where such systems are being used. This laboratory allows teachers and students to participate in its further development.

\section{Educational and Research Activities within this Laboratory}

Both the mechanical and control aspects of the laboratory's technological section have been analyzed so far. The technological section is a rather complex mechatronic system. When parking, it is necessary to avoid injury to persons or damage to vehicles. Therefore, the ATC technology is equipped with sophisticated sensors and control systems. The following steps must be performed in sequence: First, drive the car close to the front of the gate and open the outer gate by touching the smart card to the card reader attached to the gatepost. Then, drive the car on the pallet, put the handbrake on and leave the car in gear. After that, get out of the car and leave the check-in area. Finally, start the parking sequence by retouching the smart card to the card reader.

The parking sequence is divided into the following steps:

1. When the conditions checked and indicated on the information panel (vehicle size and weight) are met, the car is fixed to the pallet with linear actuators.

2. The fixed pallet, which is attached to the slewing unit, rotates 90 degrees in the direction of the first floor's ready skip.

3. After rotating the pallet and opening the inner gate, the pallet on the slewing unit is locked off and the skip's lift arm is released. The lift arm catches the pallet with the car and moves it to the skip.

4. The skip then moves to a designated parking spot on the first floor or change its position to reach the level of the lifting device (elevator).

5. Using the lift arm, which releases the pallet with the car to the opposite side, the car is moved to the elevator area.

6. The elevator brings the car to the designated floor where it is to be parked.

7. The ready skip on the particular floor, which is intended for moving the car on the pallet within the floor, releases its lift arm to catch the pallet in the elevator and move it to skip.

8. Once the car is positioned on the skip, the car is transported towards the designated parking spot. 
9. The lift arm then moves the car to the parking spot.

10. Simultaneously with these steps, an empty pallet from another parking spot is moved to the slewing unit.

The heart of the system which controls the automatic operation of the parking sequence is a Siemens SIMATIC ET 200S PLC located in the switchboard of the technology's stationary section. Via a Wi-Fi network, this controller configures autonomous tasks which are performed on transport carts (skips) by a SIMATIC S7-1200 PLC.

Students are not allowed to interfere in the control algorithms in these PLCs so that the safe operation of the entire system is protected from being endangered. To visualize details of the entire parking sequence, which is subject to the conditions of dozens of sensors within the system, an application created in the Control Web development environment is used. This application assumes all information about the running process from an OPC server which runs on the same PC.

In addition to this basic automatic mode visualization, the system includes an operator's console in the form of a tablet which allows for turning off automatic mode and, in service mode, performing the parking sequence in a step-by-step manner.

In class, students can program their applications that visualize the entire process based on the values retrieved from the OPC server.

\section{Scada/HMI Visualization}

Thanks to the generosity of the Czech company GEOVAP, we managed, on very favorable terms, to equip the laboratory with their software product - the Reliance 4 SCADA/HMI system. Reliance is a professional SCADA/HMI system designed for the monitoring and control of various industrial processes and for building automation. Data is acquired from control or telemetry systems, logged to databases, and presented to end users in a graphical form (schemes, charts, tables, etc.).

Students will be assigned a number of tasks (projects) that will allow for visualizing and storing information from running parking sequences. Students will be able to carry out these tasks without endangering the safe operation of the entire process. Their applications will be based on reading data from a running OPC server. According to the results of the analysis of the system structure and parking sequences, they will visualize these sequences.

A complete application can be easily available to remote users. Reliance 4 Web Client is a Java-based program designed to run a visualization project over the Internet. Reliance 4 Smart Client is designed for use with smartphones and tablets running iOS, Android, Windows Phone, or BlackBerry OS. Students will thus be able to get acquainted with all these technologies while developing their applications.

Creating a visualization project is substantially accelerated by a well-arranged manager and wizard system. The basic features can be configured with no need 
for programming. Thanks to this, students can achieve significant time savings that can later be invested in creating further applications and their productivity should therefore increase rapidly with each newly created application.

To reliably detect errors and inconsistencies in an application, Project Diagnostics can be used. It is a tool built into the development environment that not only warns students of invalid links, non-existent tags, and syntax errors, but it also discovers unused tags. The results are displayed in a well-arranged list from which it is possible to access the respective property with just a mouse-click and fix the problem.

\section{Concept of PIL Model}

\subsection{Motivation}

PIL is a technique for a rapid prototyping when mathematic model is running in real-time, the control system is running on the target platform [2].

During the processor-in-the-loop (PIL) phase, the control is compiled and downloaded into an embedded target processor and communicates directly with the plant model via standard communications such as Ethernet. In this case, no $\mathrm{I} / \mathrm{O}$ devices are used for the communication.

The scheme of PIL model is represented by Fig. 2 and it consists of 3 blocks:

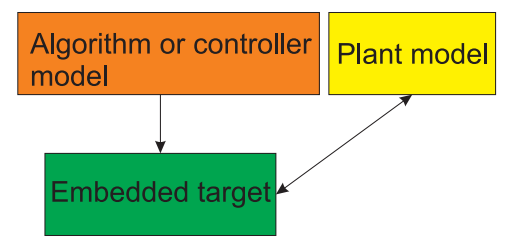

Fig. 2. Block scheme of PIL concept

The following conditions must be followed for PIL approach:

1. Mathematic model is running in real-time

2. Control system is running on the target hardware platform

3. No input/output devices are used, a data connection is used to exchange data between the control system and the model

It is mainly intended for testing the computing power of the target hardware platform and testing the control system by simulating machine malfunction.

\subsection{Design of PIL model for ATC}

Control process of the technology is performed by a distributed control system which contains several PLC communicating over the deterministic protocol 
PROFINET. The main PLC is SIMATIC S7 300 and it communicates with the other PLCs (S7 1200) located in the center of each floor of the object. Furthermore, the technology contains two I/O modules and drive converters.

Due to the PIL concept, all of the signals from PLCs, I/O modules and drive converters are subject to PIL simulation and thus must be re-mapped to match the PROFINET protocol standard with the aim of establishing communication with the "slave" side of the system that is ALIX PC with PROFINET slave card CIFX90-RE $\backslash F$.

The particular structure of PIL model of ATC is represented by Fig. 3. It contains the following parts:

1. Control system on target hardware

It is embedded target with the current control system. Usually it is the original control system with no changes. For the purposes of this project we used the identical copy of the control system except I/O modules that have been emulated (re-mapped) to profinet communication while the control algorithms of course stay unchanged.

2. Mathematical model (CPU+HW)

This the CPU declared by the definition of PIL model. Technically it is a computer board with a slave profinet card with the running real-time target, particularly the REX Control System. This part (software simulation) emulates the real technology and thus it has to act in the same way.

\section{Host PC}

Any remote PC connected by the Ethernet. It can serve for design and implementation of mathematical model, SCADA/HMI visualization, diagnostic and remote control and of all variables in the system. Its main goal is to perform tests of behavior of the original control algorithms because unlike the real technology, the software model can represents any states of the system that can occur, including possible dangerous constellation representing highly non-standard, unlikely or impossible situations.

Mathematical models of the particular technology units can be created by means of function blocks of the REX Control System. It is composed of many blocks for various purposes: configuration of real-time executive, processing of analogue signals, logic control, dynamic systems simulation, continuous control, math blocks, data archiving, and input/output blocks for reading/writing data to the real I/O modules matching the chosen hardware platform. There are two main applications for design and compiling of the control scheme (RexDraw) and diagnostic graphic tool (RexView) to upload the program, access the variables and trends. However, the detailed description of the REX Control System and its all features and capabilities is beyond the scope of this paper and all the relevant information can be found in [3]-[5].

Fig. 4 shows the example of software model for a part of the system, particularly for motors and skips. It consists of two parts that is executive and main task. An example of implementation of control structure is mentioned in [6].

It presents the way how the I/O data is read and written by so called "flags" from/into the PROFINET slave (Simatic PLCs). The particular flags represents 


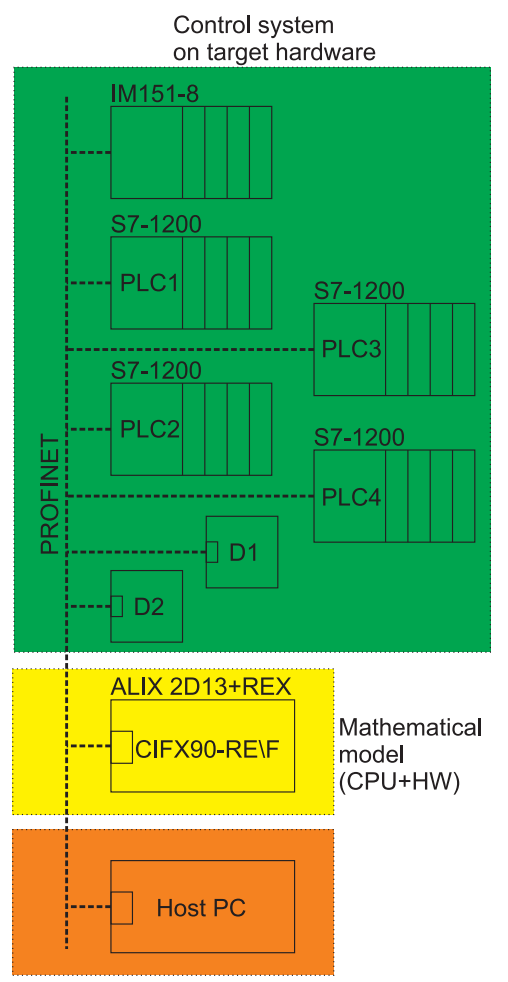

Fig. 3. PIL model of Automated Transportation Center in Ostrava

step-by-step the signals defined in the original control system based on PLCs, while the definition is read from HW configuration of the PLC.

Hence, by use of standard or user-defined software blocks, it is possible to process the signals according users' needs and requirements, see the screenshot of RexView utility in Fig. 5. Typically, it helps to simulate the most common sequences in the system with the aim of either optimization or finding critical or weak links in the system during putting a car inside the ATC and out [7], [8].

\section{$5 \quad$ Features and Novelty of the Solution}

The main objective of using PIL model for Automated Transportation Center was to implement possibility to carry out various load tests of the designed control system performed by PLCs that control real technology. Unlike HIL model (hardware-in-the-loop), there is no need to use real i/o modules as all the signals are re-mapped for PROFINET protocol. Also, due to the large number of the signals, this approach helped to save up costs significantly.

The novelty of the solution lies in the use of a hard real-time target for the plant model of the complex technology that offers complex possibilities regard- 


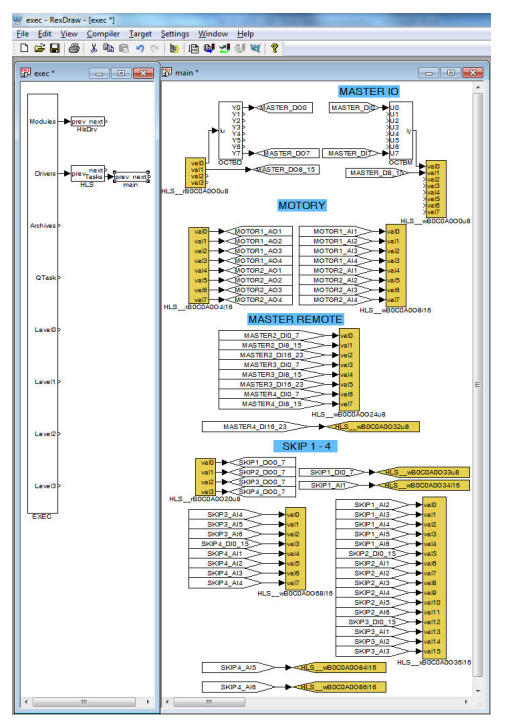

Fig. 4. Example of the source scheme in REX Control System (RexDraw)

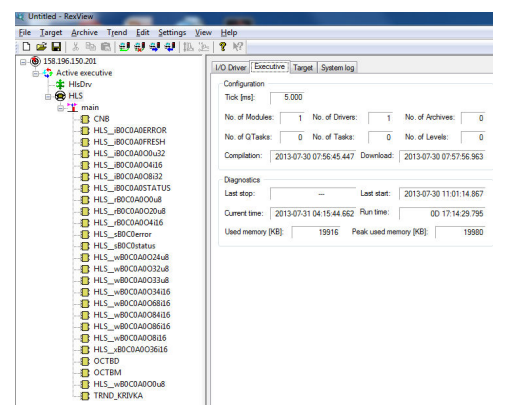

Fig. 5. A view of the diagnostic and visual utility (RexView)

ing processing input/output signals, mainly powerful libraries for mathematical operations, regulation, archiving and others. Of course there are more similar solutions on the market, but not many of them follow the needs of hard real-time and low-cost budget or at least a good performance/costs ratio. The main reasons for use of the REX Control System are very good price and multiplatform support, see Fig. 6. Besides, it is compatible with the Matlab\&Simulink platform, thus the control algorithms can be fully simulated before deployment to the target. It supports industrial OPC standard, visualization on the web through HTML5. It offers development of new function blocks (written in $\mathrm{C}$ language), use of a vast number of i/o drivers (for different hardware platforms, distribu- 
tors and third- party products), and possibility to port to various operating system platforms (for example Windows CE, Windows Embedded, Linux/Xenomai, PharLap ETS, Windows7/Vista/XP.

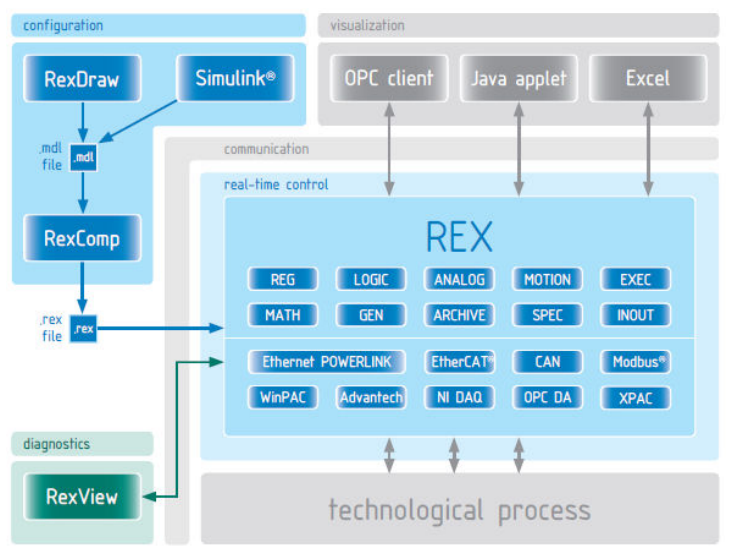

Fig. 6. Basic overview of REX control system configuration

\section{Testing the Operation of PIL Model}

This chapter briefly describes results of testing of two chosen case studies: outer doors and rotary unit.

\subsection{Outer Doors}

At the beginning of the experiment the outer doors are closed (down), see Fig. 7 (LY=true). This is in accordance with the overview of SCADA/HMI visualization. Once the Open button is clicked, the moving of outer doors is simulated by integrating the input signal to the integrator block, see visualization screenshot in Fig. 8 where both "Outer doors open" and "Outer doors closed" indicators are grey (false value), corresponding to false values of and LY and HY in Fig. 9 - doors are not either down nor up. After predefined time the doors reach the final upper position, as indicated in Fig. 10 in visualization (green indicator) screenshot and in Fig. 11 in REX control system screenshot.

\subsection{Rotary Unit}

At the beginning of the experiment the rotary unit is in its right position, as indicated in Fig. 12. Once the model received the command to rotate, the PIL 


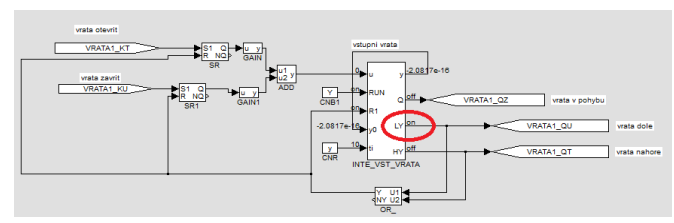

Fig. 7. Start of experiment in REX: outer doors closed (down)

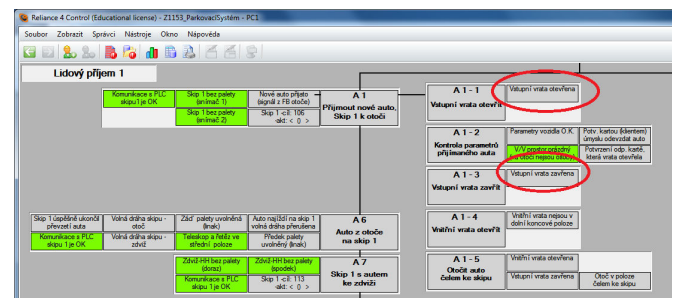

Fig. 8. Progress of the experiment in visualization: outer doors moving

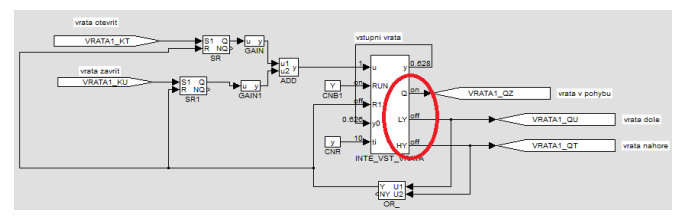

Fig. 9. Progress of the experiment in REX: outer doors moving

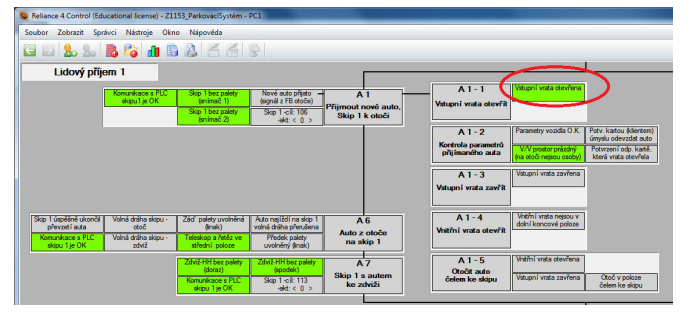

Fig. 10. End of experiment in visualization: outer doors open (up)

model starts bidirectional communication with PLC distributed system to handle all control and states concerning the frequency drives. When all other conditions are followed to enable rotary unit to slide (for example release of blockade of the rotary unit and others), the moving of rotary unit is simulated by integrating the input signal to the integrator. After predefined time the output signal reaches 


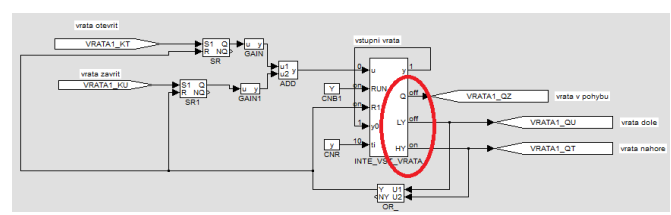

Fig. 11. End of experiment in REX: outer doors open (up)

the final value that means rotary unit finished its moving and came to its left position, see Fig. 13.

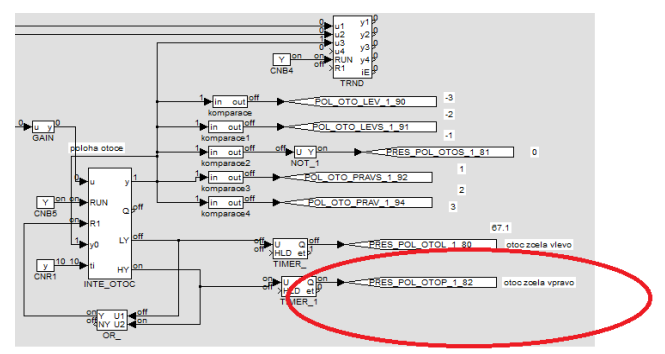

Fig. 12. Start of experiment in REX: rotary unit on the right position

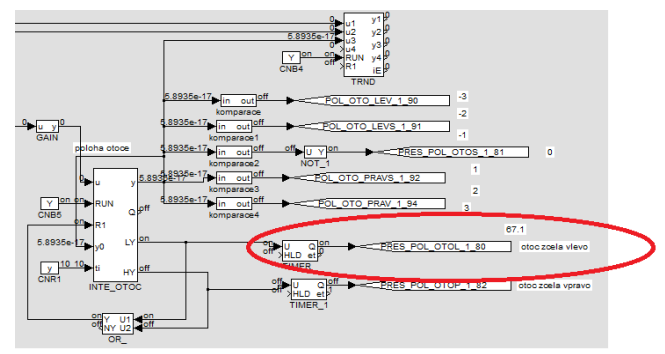

Fig. 13. End of experiment in REX: rotary unit on the left position 


\section{Conclusion}

The main idea of the presented paper is to describe the concept of Automated Transportation Center and the use of the REX Control System for processor-inthe-loop model.

At present day, the PIL communication between physical control system and CPU has been established over the PROFINET network. Due to the security reasons, identical copy of the PLC CPUs have been used to work with the PIL model (1x IM 151-8, 4x S7-1200). Besides, the Automated Transportation Center is declared as a heavy laboratory so it will serve for educational purposes, too. Students will be able to deal with programming and visualization of typical real example of automation problematic with the use of modern software and hardware equipment. Fig. 14 shows the plant model of the technology implemented on computer ALIX 2D13+REX Control System under Linux OS with CIFX90-RE- $\backslash$ F PROFINET Slave card in mini-PCI Express.

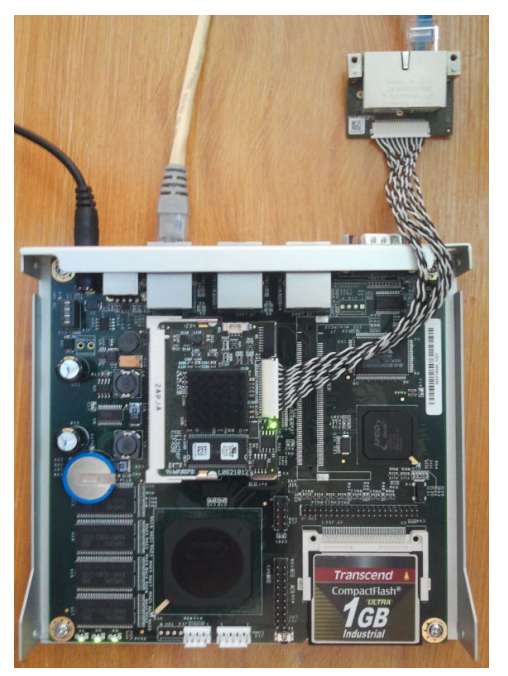

Fig. 14. PIL model of the technology of ATC

The software blocks representing all of the particular technology units (lift, skips, and the others) are newly implemented at the moment and it is currently under tests.

Existing experience confirm the correctness of technical solution. The main contribution of the approach is the fact that configuration of the PIL model with the REX Control System can be applied not only for particular Siemens PLCs with the PROFINET communication but also for a general control system based on different brands of PLCs communicating over some of the standard communication protocols. 
The objective approach belongs to the group of techniques generally referred to as "Model based design" and recently intensively developed by many software companies, for example by MathWorks as a part of Matlab\&Simulink real-time tools.

Acknowledgments Research supported by project SP2014/156, "Microprocessor based systems for control and measurement applications." of Student Grant System, VSB-TU Ostrava.

\section{References}

1. VysokeSkoly.cz, Automatizované dopravní centrum Ostrava na VŠB-TUO, http: //www.vysokeskoly.cz/clanek/novy-clanek-5309

2. Francis, G., Burgos, R., Rodriguez, P., Wang, F., Boroyevich, D., Liu R., Monti, A.: Virtual prototyping of universal control architecture systems by means of processor in the loop technology. In the APEC 2007: TWENTY-SECOND ANNUAL IEEE APPLIED POWER ELECTRONICS CONFERENCE AND EXPOSITION, VOLS 1 AND 2, pp. 21-27, Anaheim (2007)

3. Balda, P., Schlegel, M., Stetina, M.: Advanced Control Algorithms + Simulink Compatibility + Real-time OS $=$ REX. In: $16^{\text {th }}$ Triennial World Congress of International Federation of Automatic Control, pp. 121-126, vol. 16, Prague (2005)

4. Kocanek M., Balda, P.: General sequential function charts editor. In $12^{\text {th }}$ International Carpathian Control Conference, pp. 191-194, Velke Karlovice (2011)

5. REX Controls, ZCU Plzen, Czech Republic, http://www.rexcontrols.com

6. Ozana, S., Pies, M., Slanina Z., Hajovsky, R.: Design and Implementation of LQR controller for Inverted Pendulum by use of REX Control System. In $12^{\text {th }}$ International Conference on Control, Automation and Systems, pp. 343-347, Jeju (2012)

7. Kocian, J., Tutsch, M., Ozana S., Koziorek, J.: Application of Modeling and Simulation Techniques for Technology Units in Industrial Control. In Lecture Notes in Electrical Engineering, 3rd International Conference on Computer, Communication, Control and Automation, 3CA 2011, Zhuhai (2011)

8. Kocian, J., Tutsch, M., Ozana S., Koziorek, J.: Modeling and Simulation of Controlled Systems and Technologies in Industrial Control. In ICACTE 2011: Proceedings of the $4^{\text {th }}$ International Conference on Advanced Computer Theory and Engineering, ASME Press, pp. 213-217, New York (2011) 